\title{
Are we missing opportunities for early intervention in bipolar disorder?
}

Expert Rev. Neurother. 12(1), 5-7 (2012)

\author{
Craig A Macneil \\ Author for correspondence: \\ Orygen Youth Health and Research Centre, \\ Melbourne, Australia \\ Tel: +61393422800 \\ Fax: +61393873003 \\ craig.macneil@mh.org.au
}

\section{Karen Hallam}

Deakin University, Geelong, Australia and

Department of Psychology, Victoria University,

Melbourne, Australia

\section{Philippe Conus}

Orygen Youth Health Research Centre, Centre for Youth Mental Health, University of Melbourne,

Melbourne, Australia

and

Treatment and Early Intervention in Psychosis Program (TIPP), Département de Psychiatrie CHUV,

Université de Lausanne, Clinique de Cery, 1008 Prilly, Switzerland

\section{Lisa Henry}

Orygen Youth Health Research Centre, Centre for Youth Mental Health, University of Melbourne, Melbourne, Australia

\section{Linda Kader}

Orygen Youth Health and Research Centre, Melbourne, Australia

\section{Michael Berk}

Orygen Youth Health Research Centre, Centre for Youth Mental Health, University of Melbourne, Melbourne, Australia

and

Deakin University, Geelong, Australia

and

Mental Health Research Institute, Melbourne, Australia

\author{
"The staging model ... provides the opportunity for specialized \\ and stage-appropriate interventions that may minimize the risk \\ for further illness progression, and facilitate recovery earlier in \\ the course."
}

Clinical staging informs the assessment, prognosis and choice of therapies in a diverse range of medical specialities, such as cardiac disorders and oncology. However, it is only relatively recently that psychiatry has begun to explore staging as a conceptual framework to understand the progression of psychiatric disorders, and how this may inform prognosis and management choices. However, a substantial body of new data is available to inform this topic. Therefore, it appears timely to examine some of the evidence regarding early intervention and the staging of clinical approaches, specifically as they relate to bipolar disorder.

Considerable evidence has emerged in recent years for a staging model in medicine, particularly for disorders such as cancer, HIV and liver disease. According to this model, disorders progress in identifiable phases, which have specific features and require specifically adapted interventions. There is recognition that while progression through various phases may not be applicable to all patients with a particular disorder, this concept can nevertheless reflect an aggregate picture.

The staging model has also attracted interest in psychiatry as it provides the opportunity for specialized and stageappropriate interventions that may minimize the risk for further illness progression, and facilitate recovery early in the course. Specifically, recent models developed for psychotic disorders and later adapted to bipolar disorders, have described categories ranging from stage 0 (describing and identifying risk factors for a disorder in the absence of any clear symptoms), through to stages $1 \mathrm{a}$ and $1 \mathrm{~b}$ (comprising mild, nonspecific identifiable prodromal symptoms, respectively), stage 2 (comprising the first episode), stages $3 \mathrm{a}, 3 \mathrm{~b}$ and $3 \mathrm{c}$ (comprising subthreshold, threshold and persistent relapse, respectively), to stage 4 (referring to persisting unremitting symptoms that may have been nonresponsive to treatment) [1-3].

Of considerable interest in recent years, and as a significant cornerstone of the staging model, is the concept of neuroprogression. This concept has its roots in the work of Post and colleagues, who posited the concept of neurosensitisation, or 'kindling' in 1986 [4]. Our understanding of the neurobiological mechanisms that may underpin this phenomenon have shifted from an early focus on monoamines, and has become successively broader. Specifically, cortisone, oxidative, mitochondrial and inflammatory cytokine mechanisms are gaining more credibility as models of understanding treatment nonresponsiveness with the progression of psychiatric disorders, and bipolar disorder in particular [5]. The shared mechanism of action may also go some way to explaining the effectiveness of medications as apparently disparate as lithium, valproate and antipsychotic medications, which have been found to increase brainderived neurotrophic factor in animal models [6], and share many effects on neurogenesis, apoptosis, inflammatory pathways and oxidative biology. 
There are a number of reasons why a staging approach may be appropriate and clinically effective. Perhaps most notably, for some time it has been recognized that psychiatric disorders can present with a progressive course, with Kraepelin having observed that periods of wellness between relapses in bipolar disorder often become shorter with each episode [7]. This finding, in addition to evidence that bipolar disorder is characterized by progressive neuropathological processes, has been replicated by a significant volume of subsequent research [5].

${ }^{66} \mathrm{~A}$ significant aspect of early intervention in bipolar disorder is that it may provide a 'window of opportunity' to prevent, or at least minimize, some of the secondary morbidity that can occur with progressive episodes."

Specifically, and of considerable clinical importance, neuroimaging data are emerging showing that the hippocampal volume loss and ventricular dilatation that occur early after the first episode of schizophrenia may progress with subsequent relapses in bipolar disorder, and that for bipolar disorder, gross brain structure is relatively preserved in the early phase [8]. Consequent to this, there is some evidence that there are progressive neuropsychological changes with the course of the disorder [9]. Clearly, therefore, there is a significant incentive to attempt to prevent the potentially deteriorating course of bipolar disorder through early identification and provision of phase-appropriate treatment. This improved rationale for early and appropriate intervention allows the clinician to work with the patient to actively prevent progression through pharmacological and psychological approaches.

Regarding intervention itself, the first step needs to be that of accurate diagnosis. It is widely recognized that bipolar disorder is often initially misdiagnosed, with a large study reporting that $69 \%$ of people with bipolar disorder described being misdiagnosed at least once [10]. In addition, a latency period of over 12 years between initial onset of symptoms and commencement of treatment is not uncommon [11]. However, useful reviews describing differentiating symptoms that may assist in early detection and diagnosis have been reported [11].

Research has focused on the efficacy of specific therapies at various phases in the course of bipolar disorder. Studies by Gelenberg et al. [12] and Swann et al. [13] indicated that the effectiveness of lithium may be reduced with successive episodes. More recently, Berk et al. examined pooled data of 4346 participants from 12 studies in order to establish the efficacy of an atypical antipsychotic in the treatment of bipolar disorder [14]. Using measures of mania, depression, overall global impression and relapse, it suggested that individuals at the earliest stages of illness consistently had a more favorable response to treatment. In the Systematic Treatment Enhancement Program for Bipolar Disorder (STEP BD) database, number of episodes was robustly related to ratings of mania, depression, functioning and quality of life [15].

Regarding psychological treatments for bipolar disorder, Reinares et al. [16] and Colom [17] reported that the number of previous episodes had a significant effect on outcome following psychoeducational interventions. Similarly, Scott et al. who conducted the largest trial of cognitive-behavioral therapy for bipolar disorder to date, found clear evidence that psychological intervention was more effective in the early phase of the disorder (i.e., from episodes 1-6), and that cognitive-behavioral therapy actually led to higher relapse rates than treatment as usual for people who had experienced more than 30 episodes [18]. By contrast, a recent review by Lam et al. concluded that these interventions are effective in preventing or delaying relapse regardless of the number of previous episodes [19].

A significant aspect of early intervention in bipolar disorder is that it may provide a 'window of opportunity' to prevent, or at least minimize, some of the secondary morbidity that can occur with progressive episodes. Scott identified that, for people with bipolar disorder, multiple episodes could be associated with financial and employment difficulties, and self-esteem, guilt, loss and adjustment issues [20]. Furthermore, bipolar disorder also typically has its onset during late adolescence and early adulthood, which is widely recognized as a critical phase in an individual's psychosocial development. Therefore, interventions that focus on minimizing disruption to the developmental trajectory though utilizing effective psychopharmacology and implementing psychosocial approaches, including psychoeducation, medication adherence, relapse prevention, social recovery and assisting adaptation to the disorder, have considerable face validity.

\section{6 [Staging] provides an optimistic framework for employing optimal biological and psychosocial interventions in the early phases of bipolar disorder."}

Functional recovery is also becoming an area of increased importance and interest. Much of the literature concurs that while approximately $90 \%$ of people achieve symptomatic recovery following an episode of bipolar disorder, only approxiamtely $40 \%$ obtain satisfactory social and occupational recovery [21,22]. This disparity, in addition to the importance of assisting people maintain social networks following a first episode of bipolar disorder, has been described elsewhere [23], and should clearly be a focus of intervention. Again, through the emerging understanding of the neuroprotective properties of some medications, supplements and psychosocial interventions, there may be an increased likelihood to improve functional recovery early, which is subsequently associated with better trajectory of the disorder and prognosis.

In conclusion, biological and psychological research on bipolar disorder provides support for a staging model in which specific interventions should be tailored to the phase of the disorder. This provides an optimistic framework for employing optimal biological and psychosocial interventions in the early phases of bipolar disorder as they could prevent the secondary consequences of the illness, and might be neuroprotective and prevent or minimize stage progression and some of the neurobiological, psychological, functional and social morbidity that can occur in the context of repeated episodes. This 
requires early and accurate identification and diagnosis, in addition to pharmacological and psychosocial interventions designed to prevent both primary and secondary morbidity.

\section{Financial \& competing interests disclosure}

CA Macneil is funded part-time by Orygen Youth Health Research Centre, Centre for Youth Mental Health, University of Melbourne (Melbourne,
Australia). There are no conflicts of interest for this editorial. The authors have no other relevant affiliations or financial involvement with any organization or entity with a financial interest in or financial conflict with the subject matter or materials discussed in the manuscript apart from those disclosed.

No writing assistance was utilized in the production of this manuscript.

\section{References}

1 McGorry PD, Hickie IB, Yung AR et al. Clinical staging of psychiatric disorders: a heuristic framework for choosing earlier, safer and more effective interventions. Aust. NZ J. Psychiatry 40, 616-622 (2007).

2 Berk M, Hallam K, Malhi G et al. Evidence and implications for early intervention in bipolar disorder. J. Mental Health 19(2), 113-126 (2010).

3 Kapczinski, F, Dias VV, Kauer-Sant'Anna $\mathrm{M}$ et al. Clinical implications of a staging model for bipolar disorders. Expert Rev. Neurother. 9, 957-966 (2009).

4 Post RM, Rubinow DR, Ballenger JC. Conditioning and sensitisation in the longitudinal course of affective illness. Br. J. Psychiatry 149, 191-201 (1986).

5 Berk M, Kapczinski F, Andreazza AC. Pathways underlying neuroprogression in bipolar disorder: focus on inflammation, oxidative stress and neurotrophic factors. Neurosci. Biobehav. Rev. 35, 804-817 (2011).

6 Martinowich K, Manji HK, Lu B. New insights into BDNF function in depression and anxiety. Nat. Neurosci. 10, 1089-1093 (2007).

7 Kraepelin E. Lifetime Editions of Kraepelin in English (Volume 5). Thoemmes Press, Bristol, UK (2002).

8 Lisy ME, Jarvis KB, DelBello MP et al. Progressive neurostructural changes in adolescent and adult patients with bipolar disorder. Bipolar Disord. 13(4), 396-405 (2011).
9 López-Jaramillo C, Lopera-Vásquez J, Gallo A et al. Effects of recurrence on the cognitive performance of patients with bipolar I disorder: implications for relapse prevention and treatment adherence. Bipolar Disord. 12(5), 557-567 (2010).

10 Hirschfeld RM, Lewis L, Vornik LA. Perceptions and impact of bipolar disorder: how far have we really come? Results of the National Depressive and Manic Depressive Association 2000 survey of individuals with bipolar disorder. J. Clin. Psychiatry 64 , 161-174 (2003).

11 Berk M, Dodd S, Callaly P et al. History of illness prior to a diagnosis of bipolar disorder or schizoaffective disorder. J. Affect. Dis. 103, 181-186 (2007).

12 Gelenberg AJ, Kane JN, Keller MB et al. Comparison of standard and low serum levels of lithium for maintenance treatment of bipolar disorders. N. Engl. J. Med. 321, 1489-1493 (1989).

13 Swann AC, Bowden CL, Calabrese JR, Dilsaver SC, Morris DD. Differential effect of number of previous episodes of affective disorder on response to lithium or divalproex in acute mania. Am. J. Psychiatry 156(8), 1264-1266 (1999).

14 Berk M, Brnabic A, Dodd S et al. Does stage of illness impact treatment response in bipolar disorder? Empirical treatment data and their implication for the staging model and early intervention. Bipolar Disord. 13, 87-98 (2011).

15 Magalhães PV, Dodd S, Nierenberg AA, Berk M. Cumulative morbidity and prognostic staging of illness in the
Systematic Treatment Enhancement Program for Bipolar Disorder (STEP-BD). Aust. NZ J. Psychiatry (2011) (In press).

16 Reinares M, Colom F, Rosa, AR et al. The impact of staging bipolar disorder on treatment outcome of family psychoeducation. J. Affect. Dis. 123(1-3), 81-86 (2010).

17 Colom F. Long-term follow up of psychotherapies in bipolar disorder. Bipolar Disord. 10(Suppl. 1), 20 (2008).

18 Scott J, Paykel E, Morriss R et al. Cognitive behavioural therapy for severe and recurrent bipolar disorders: randomised controlled trial. Br. J. Psychiatry 188, 313-320 (2006).

19 Lam DH, Burbeck R, Wright K, Pilling S. Psychological therapies in bipolar disorder: the effect of illness history on relapse prevention - a systematic review. Bipolar Disord. 11, 474-482 (2009).

20 Scott J. Psychotherapy for bipolar disorder. Br. J. Psychiatry 167 (5), 581-588 (1995).

21 Strakowski SM, Williams JR, Fleck DE, Delbello MP. Eight month functional outcome from mania following a first psychiatric hospitalization. J. Psychatric Res. 34 (3), 193-200 (2000).

22 Tohen M, Hennen J, Zarate CM et al. Two year syndromal and functional recovery in 219 cases of first episode major affective disorder with psychotic features. Am. J. Psychiatry 157 (2), 220-228 (2000).

23 Conus P, McGorry PD. First episode mania: a neglected priority for early intervention. Aust. NZ J. Psychiatry 36, 158-172 (2002). 